\title{
CHROMATIC DISCRIMINATION UNDER DIFFERENT STATES OF CHROMATIC ADAPTATION
}

\author{
Urbin, Á., Nagy, B.V., Wenzel, K. \\ Department of Mechatronics, Optics and Mechanical Engineering Informatics, Budapest University of \\ Technology and Economics, Budapest, HUNGARY \\ urbin@mogi.bme.hu
}

DOI 10.25039/x44.2017.PP02

\begin{abstract}
The objective of our study was to examine the effect of different states of chromatic adaptation on the adapted white point based on chromatic discrimination measurements.

In the study normal colour observers performed the trivector test of the Cambridge Colour Test (CCT) in order to measure the just-noticeable stimuli on the three confusion lines under different states of chromatic adaptation. Two states of chromatic adaptation were achieved applying colour filters. The tests were accomplished without filter as a reference.

The factors (and their levels) of the statistical analysis were the followings: state of adaptation (no filter and the two different filters), analyzed confusion line (Protan, Deutan and Tritan), and background chromaticity (distance from the neutral point towards the chromaticity point of the filter and in the opposite direction).

The analysis shows that adapting to the filters affects colour discrimination depending on the background chromaticity compared to the results with the reference test. In our results we estimate the change in chromaticity of the background for best colour discrimination.
\end{abstract}

Keywords: Chromatic discrimination, Chromatic adaptation, Just-noticeable stimuli, Cambridge Colour Test (CCT)

\section{Introduction}

The experiment introduced in this paper is part of a research that aims to understand the limits and the mechanisms of chromatic adaptation based on differences and changes in chromatic discrimination abilities of normal colour observers under different states of chromatic adaptation.

\section{Measurements}

The method of observing chromatic discrimination is measuring the just-noticeable stimuli. The smaller this stimuli are, the better resolution we can assume in chromatic discrimination.

In this paper the results of two experiments are described. One set of measurements with coloured filters in which 18 subjects participated and one set of measurements with unfiltered eyes with the participation of 25 subjects considered as reference. Subjects of both experiments were between 20 and 22 years old, each of them normal colour observer. In both experiments subjects performed the trivector test of the Cambridge Colour Test (CCT).

The measurements were executed in the Visual Systems Laboratory at the Department of Mechatronics, Optics and Mechanical Engineering Informatics, Faculty of Mechanical Engineering, Budapest University of Technology and Economics.

\subsection{States of chromatic adaptation}

The different states of chromatic adaptation were accomplished by applying two coloured filters: a purple one and a green one (further on F1 and F2 respectively). The spectral transmission of the filters can be seen on Figure 1. Measurements with unfiltered eyes will be denoted further on as NF (No Filter). 


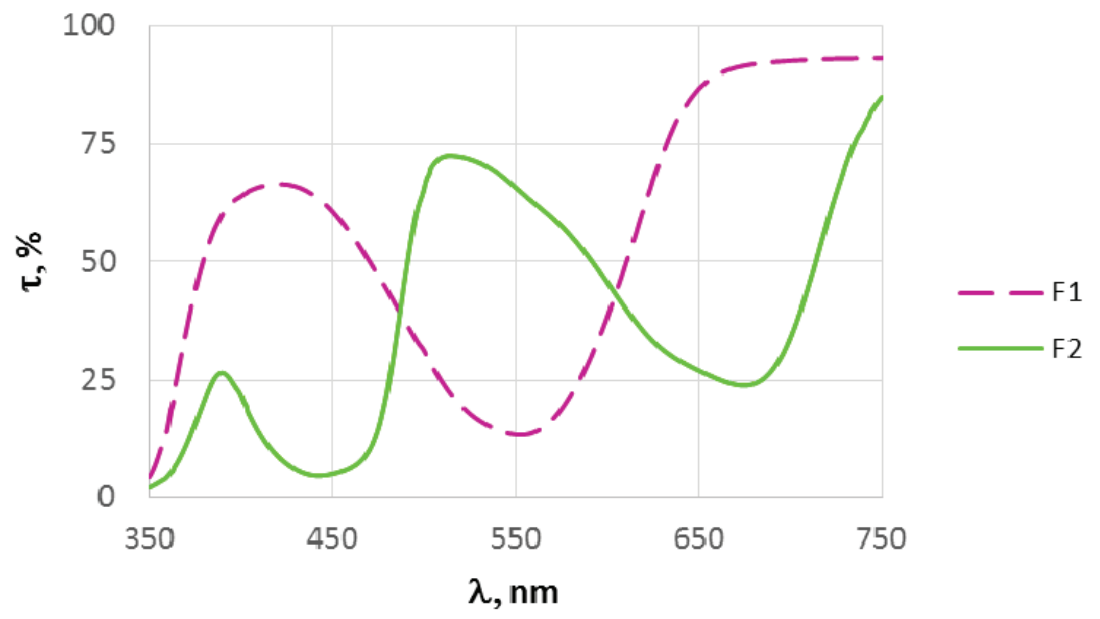

Figure 1 - Spectral transmission of the applied filters

Measurements were carried out in a dark room. Before starting the measurements subjects were asked to wear the lenses until they can judge a white surface to be achromatic. Wearing $\mathrm{F} 1$ this took 5-6 minutes however wearing $\mathrm{F} 2$ we maximized this period in 10 minutes as none of the subjects reported full adaptation and the white surface remained greenish.

\subsection{Cambridge Colour Test (CCT) - trivector test}

Cambridge Colour Test is a display-based pseudoisochromatic test (Hasrod et al., 2015., Regan et al., 1994.) that provides the just-noticeable stimuli as result in defined directions from a reference point in the CIE 1976 UCS diagram (Schanda, 2007.). Subjects performed the trivector test in which three directions are defined towards the three confusion points. Reference points defined the chromaticity of the background while the chromaticity of the Landolt-C pattern changed on the three confusion lines in order to find the three thresholds.

\subsubsection{Luminance levels}

Display luminance levels were set in order to avoid measuring the effect of differences in luminance instead of differences in chromaticities between filters as seen in Table 1. The luminance values were equalized based on the spectral transmission of the filters weighted with the $V(\lambda)$ spectral luminous efficiency function.

Table 1 - The minimum and maximum of luminance levels of the displayed pseudoisochromatic plates set for measurements with unfiltered eyes (NF) and with the coloured filters (F1 and F2)

\begin{tabular}{|l|l|l|l|}
\hline & $N F$ & $F 1$ & $F 2$ \\
\hline$L_{\max }\left[\mathrm{cd} / \mathrm{m}^{2}\right]$ & 8 & 20 & 14 \\
\hline$L_{\min }\left[\mathrm{cd} / \mathrm{m}^{2}\right]$ & 2 & 6 & 4 \\
\hline
\end{tabular}

\subsubsection{Confusion points}

In the experiment the confusion points were fixed to the values seen in Table 2 while the reference points were varied between measurements.

Table 2 - The chromaticity coordinates in the CIE 1976 UCS diagram of the Protan, Deutan and Tritan confusion points applied in the experiment

\begin{tabular}{|l|l|l|l|}
\hline & Protan & Deutan & \multicolumn{1}{c|}{ Tritan } \\
\hline u' $^{\prime}$ & 0,6579 & $-1,2174$ & 0,2573 \\
\hline v' $^{\prime}$ & 0,5013 & 0,7826 & 0 \\
\hline
\end{tabular}




\subsubsection{Reference points}

Reference points (the chromaticity values of the test backgrounds) were defined based on spectral data. The spectral power distribution of the neutral point was assumed to be equal to the spectral power distribution of a CCT plate in which both the background and the pattern were set to the white point in CIE 1976 UCS diagram (see Figure 2).

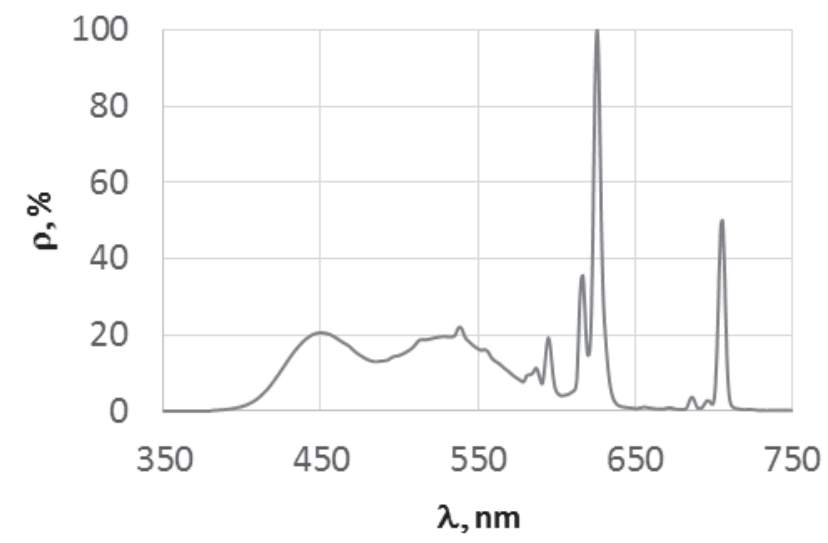

Figure 2 - Spectral power distribution of the neutral CCT plate, further on assumed as neutral white in the experiments

The second assumption was that after applying the coloured filter the neutral white point immediately appears to shift to the chromaticity point of the filter and later on during the process of chromatic adaptation this point will shift back towards the original white point.

Based on these two assumptions reference points were defined as the followings: combining the spectral power distribution of the neutral CCT plate and the transmission of the filters, two chromaticities were calculated and equidistant points $\left(\Delta \mathrm{E}_{u^{\prime} v^{\prime}}=0,0269\right)$ were set towards the neutral white point from both of these chromatic extremes (see Figure 3 ).

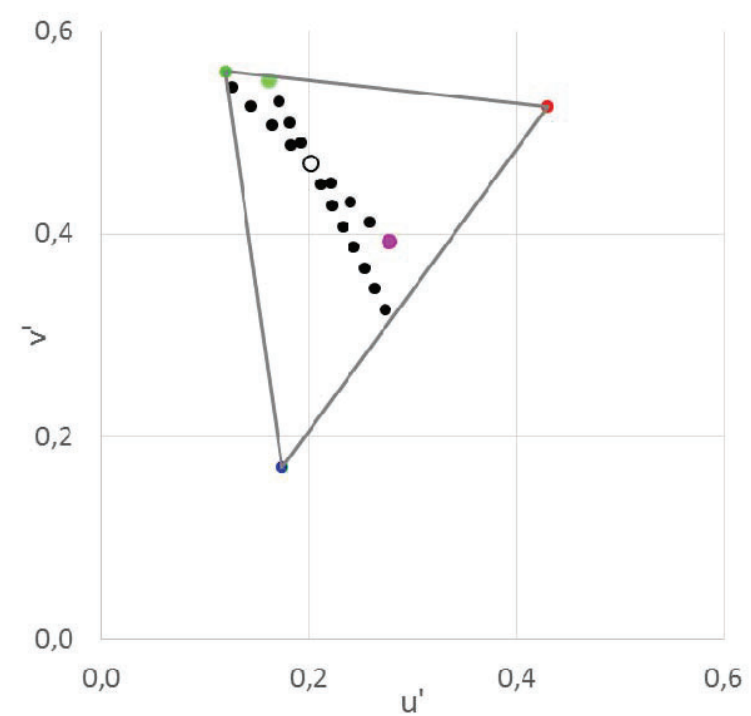

Figure 3 - The reference points set in the measurements

\section{Analysis}

The factors (and their levels) of the statistical analysis were the followings: state of adaptation (no filter and the two different filters), analyzed confusion line (Protan, Deutan and Tritan), and background chromaticity (distance from the neutral point towards the chromaticity point of 
the filter and in the opposite direction). Neutral point was labeled with zero and the ordinal numbers increased towards the arrows seen on Figure 4.

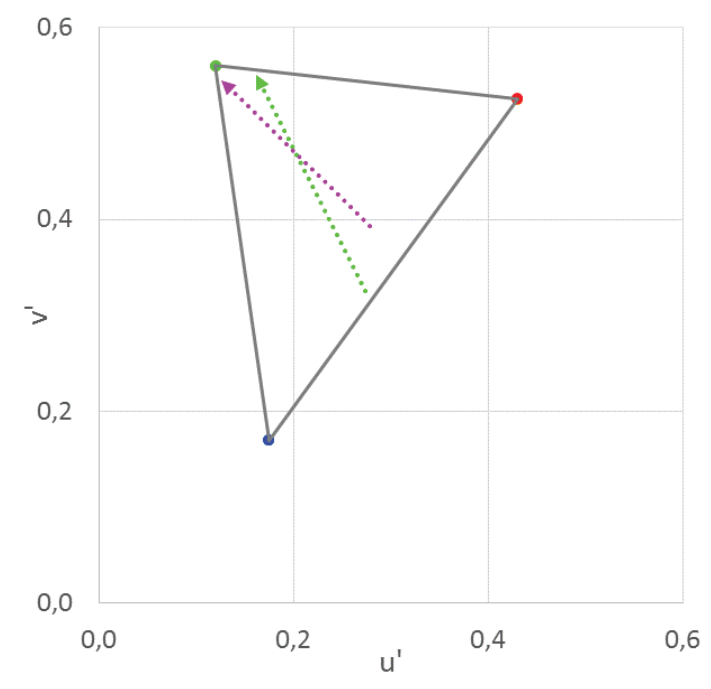

Figure 4 - The order of the reference points

The measured just-noticeable stimuli were compared using Welch's test to answer two questions detailed in the following subsections.

\subsection{Did chromatic adaptation affect colour discrimination?}

In order to find if chromatic adaptation affected colour discrimination significantly the measured just-noticeable stimuli were compared with and without filter in each reference point.

Figure 5 and Figure 6 show the results of the measurements with unfiltered eyes as a reference and with the filters (F1 and F2 respectively) towards the Protan, Deutan and Tritan confusion points in the function of reference point locus in the CIE 1976 UCS diagram. The distance of the chromaticity points from the neutral point were labeled as ' 4 ' for both filters (but in different directions as Figure 3 and Figure 4 show). The vertical axes represent the distances in CIE 1976 UCS diagram multiplied by $10^{4}$.

Green arrows mark the reference points in which the results of the measurements are not significantly different between the tests with and without filter, hence before and after chromatic adaptation to the coloured filter.

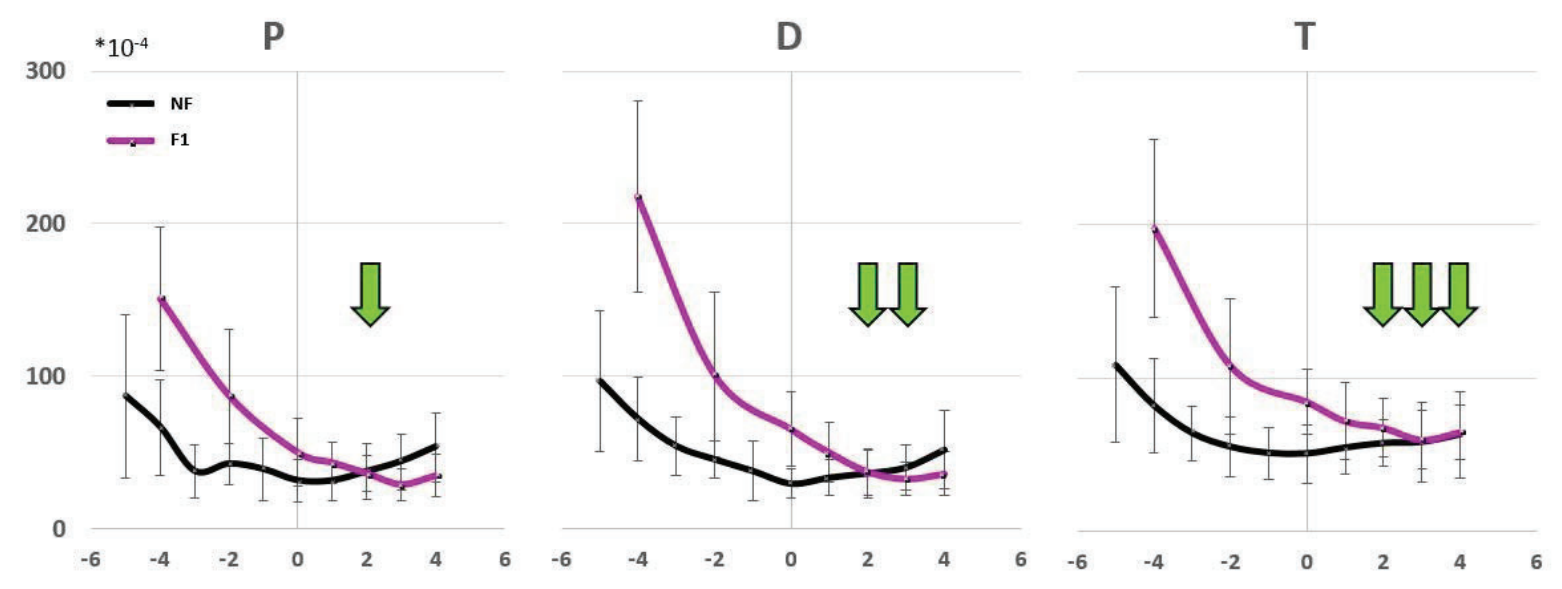

Figure 5 - Comparing just-noticeable stimuli with unfiltered eyes (NF) and with the filter F1 towards the Protan, Deutan and Tritan confusion points in the function of the reference point location in the CIE 1976 UCS diagram. 
As it can be seen on Figure 5, chromatic adaptation to F1 did affect colour discrimination on the three confusion directions similarly. The tritan direction appears to be the most stable as there were three chromaticity points in which chromatic adaptation did not show significant effect.

Figure 6 shows that adapting to $\mathrm{F} 2$ is not as clear as that to $\mathrm{F} 1$. In the tritan direction just-noticeable stimuli increase significantly and in that direction there is no measured chromaticity point in which we could say that adaptation did not have any effect. Note that there is a tendency to reach full chromatic adaptation towards the negative ordinal numbers (away from the neutral point in the opposite direction from the filter chromaticity point) that we could not measure because of the limits of our display. In the Protan and Deutan directions results show smaller differences which might be traced back to bigger inter-individual differences and to higher measurement uncertainties in general.

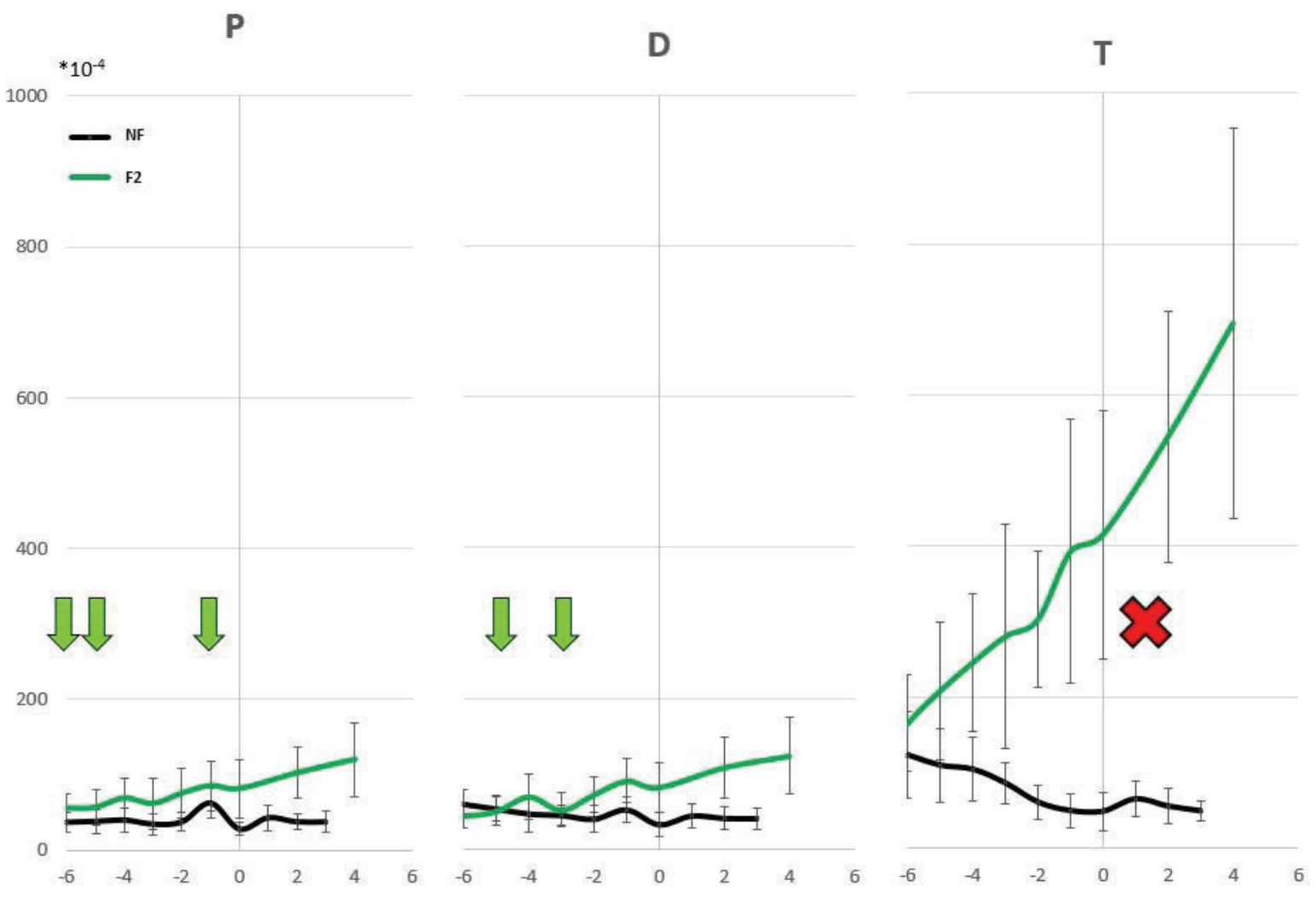

Figure 6 - Comparing just-noticeable stimuli with unfiltered eyes (NF) and with the filter F2 towards the Protan, Deutan and Tritan confusion points in the function of the reference point location in the CIE 1976 UCS diagram.

\subsection{Is the adapted white point shifted towards the observed direction?}

In order to estimate the shift of the neutral point towards the observed directions the just-noticeable stimuli with filters were compared in each reference point with the unfiltered results in the white point.

Figure 7 and Figure 8 show the results of the comparisons with the same notations as above. However, in both cases it can be stated that in the Tritan direction there is no match, it is clear from the data that the effect of F2 is much stronger than that of F1. 


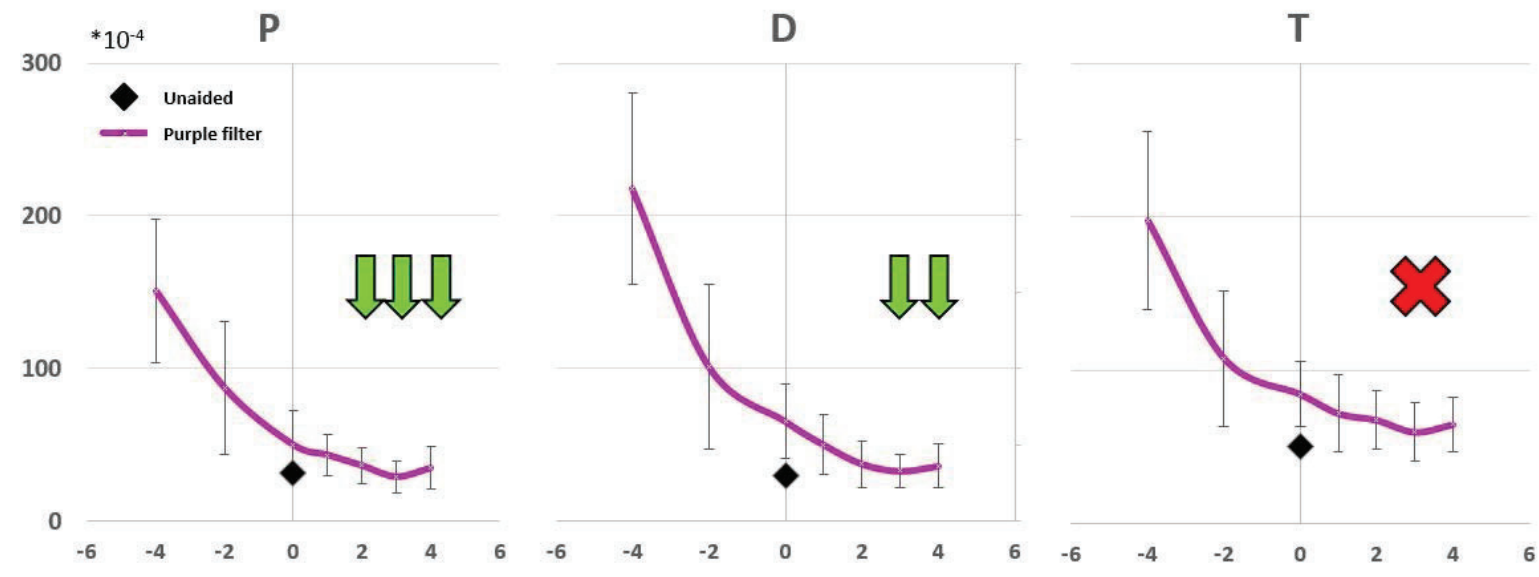

Figure 7 - Comparing just-noticeable stimuli with unfiltered eyes in the neutral point and with filter F1 in each reference point towards the Protan, Deutan and Tritan confusion points in the function of the reference point location in the CIE 1976 UCS diagram.

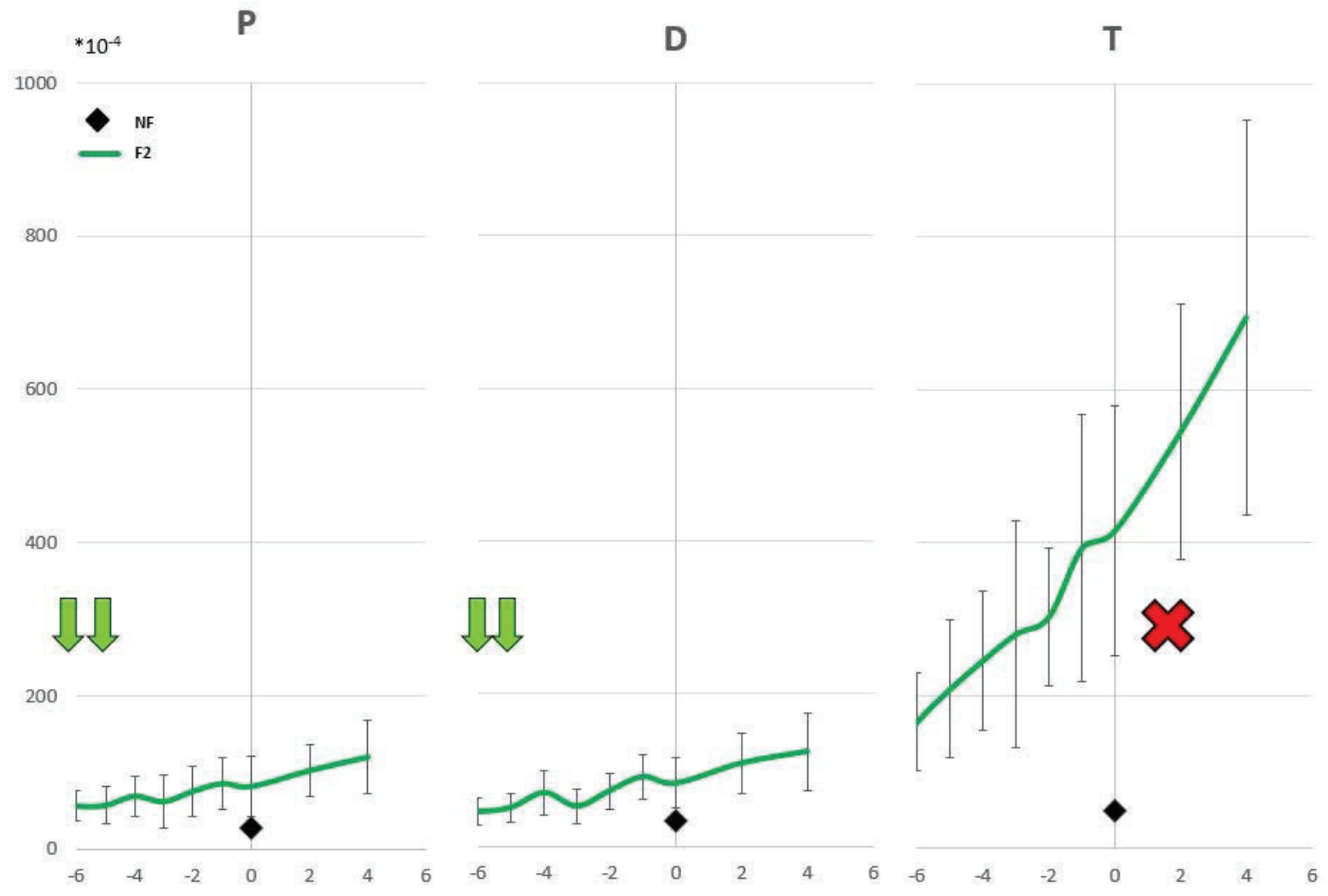

Figure 8 - Comparing just-noticeable stimuli with unfiltered eyes in the neutral point and with filter F2 in each reference point towards the Protan, Deutan and Tritan confusion points in the function of the reference point location in the CIE 1976 UCS diagram. 
Based on the matches in the Protan and Deutan directions the shift of the adapted white can be estimated as seen on Figure 9.

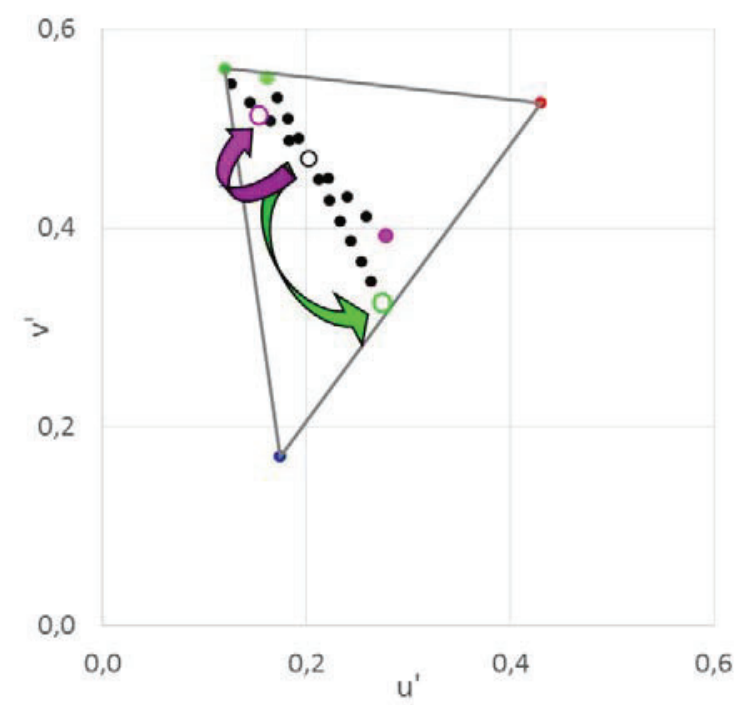

Figure 9 - Estimated shifts of the adapted white point

\section{Conclusion}

The analysis shows that adapting to the filters affects colour discrimination depending on the background chromaticity compared to the results with the reference test. In our results, we estimated the necessary change in chromaticity of the background, compensating the state of adaptation, for best colour discrimination.

Our study indicates that colour discrimination is significantly altered with changed state of chromatic adaptation at relatively small distances from the neutral point. Therefore, it might be important to compensate for its changes modifying the SPD composition reaching the adapted eye. These findings can be helpful in lighting practice where chromatic illumination is involved.

Further measurements and analysis mapping more directions in the CIE colour space are needed to describe the state of chromatic adaptation in the function of the shift of the adapted white point.

\section{References}

Color AND Vision Research LabS, 1995. Colour \& Vision Research Laboratory and Database. at http://cvrl.org, 03.08.2017.

Hasrod N., Rubin A., 2015. Colour vision: A review of the Cambridge Colour Test and other testing methods. Afr Vision Eye Health. 74(1), Art. \#23, 7 pages.

Regan, B. C., Reffin, J. P., Mollon, J. D., 1994. Luminance noise and the rapid determination of discrimination ellipses in colour deficiency. Vision Research, 34(10), pp. 1279-1299.

SCHANDA, J., 2007. Colorimetry - Understanding the CIE system. Hoboken: John Wiley \& Sons, Inc. 